\title{
OPTIMAL MULTIVARIATE GAUSSIAN FITTING FOR PSF MODELING IN TWO-PHOTON MICROSCOPY
}

\author{
Tim Tsz-Kit Lau ${ }^{1}$,Emilie Chouzenoux ${ }^{2,3}$, Claire Lefort ${ }^{4}$ and Jean-Christophe Pesquet ${ }^{2}$ \\ 1 - Department of Mathematics, The Hong Kong University of Science and Technology \\ 2 - Center for Visual Computing, CentraleSupélec, INRIA Saclay, University Paris Saclay \\ 3 - Laboratoire d'Informatique Gaspard Monge, UMR CNRS 8049, Université Paris-Est Marne-la-Vallée \\ 4 - XLIM Research Institute, UMR CNRS 7252, Université de Limoges, Limoges, France
}

\begin{abstract}
Fitting multivariate Gaussian functions constitutes a fundamental task in many scientific fields. However, most of the existing approaches for performing such fitting are restricted to 2 dimensions and they cannot be easily extended to higher dimensions. One of the main applicative areas where it is necessary to go beyond the existing techniques is the modeling of Point Spread Functions in 3D imaging. In this paper, a novel variational approach is proposed to fit multivariate Gaussians from noisy data in arbitrary dimensions. The proposed FIGARO algorithm is applied to two-photon fluorescence microscopy where its excellent performance is demonstrated.
\end{abstract}

Index Terms - PSF identification, image restoration, two-photon microscopy, proximal methods, optimization

\section{INTRODUCTION}

A large majority of biological and medical studies require the use of microscopy systems. In this context, numerous technological solutions have been developed. Among them, two-photon fluorescence microscopy (TPFM) is one of the most popular and robust techniques in fluorescence microscopy experimentally demonstrated for the first time in 1990 [1]. Based on a process theoretically predicted in 1931 [2], it has found its place owing to its specific features such as 3D imaging capabilities, micrometer resolution, or limited photobleaching thanks to the use of excitation wavelengths in the near infrared range (NIR) [3]. This nonlinear fluorescence method tends to replace classical ones working in the visible range. However, its resolution remains limited by the diffraction phenomenon, amplified by the shifting of wavelengths in NIR. In practice, the image of an infinitesimally pointsource is thus never an image-point but a spread of the infinitesimally source. The mathematical representation of the light distribution of this spread phenomenon is well-known and described by the Point Spread Function (PSF). Several super-resolution microscopy methods have been proposed to reduce the effect of the PSF, namely structured illumination microscopy (SIM), stimulated emission depletion (STED), stochastic optical reconstruction microscopy (STORM), or photoactivatable localization microscopy (PALM) (see [4] and references therein). Nonetheless, whatever the chosen imaging system, the PSF still exists which determines the optical accuracy of the microscope. The precise knowledge of the PSF pattern and its use in image processing allow access to hidden information from the raw data. In particular, image recovery techniques can be applied to the raw images in order to compensate the blur introduced by the microscope $[5,6]$. In this way, the resolution of the system can be improved and details lost during the acquisition can become visible. The implementation of an efficient deblurring strategy often requires a preliminary step of experimental data acquisition, aiming at modeling the PSF whose shape depends on the optical parameters of the microscope. The fitting model is chosen as a trade-off between its accuracy and its simplicity. Several works in this field have been inventoried and specifically developed for fluorescence microscopy $[7,8,9]$. In particular, Gaussian models often lead to both tractable and good approximations of PSF [10, 11, 12, 13, 9]. Although there exists an important amount of works regarding Gaussian shape fitting $[14,15,16,17,18,13]$, to the best of our knowledge, these techniques remain limited to the 1D or 2D cases. Moreover, only few of them take into account explicitly the presence of noise. Finally, a zero background value is usually assumed (for instance, in the famous Caruana's approach [14]). All the aforementioned limitations severely reduce the applicability of existing methods for processing real 3D microscopy datasets.

In this paper we propose a variational approach for PSF modeling through multivariate Gaussian fitting. In Section 2, the data fitting problem is formulated, and our minimization approach is introduced. The position of our contribution with respect to related works is also discussed. A convergent proximal alternating optimization method called FIGARO is then proposed to find a minimizer of the proposed cost function. In Section 3, the validity of our approach is demonstrated on 3D fluorescence bead images acquired in TPFM. Finally, Section 4 concludes the paper. 


\section{A NEW GAUSSIAN FITTING METHOD}

\subsection{Observation model}

Let us consider the problem of fitting a Gaussian model from noisy acquisitions on a $Q$-dimensional grid and let us assume that $N$ points of coordinates $\boldsymbol{X}=\left(\boldsymbol{x}_{n}\right)_{1 \leq n \leq N} \in\left(\mathbb{R}^{Q}\right)^{N}$ have been acquired. In the context of PSF modeling in microscopy, one usually has to deal with volume data (i.e. $Q=$ 3 ), and $\boldsymbol{X}$ maps the positions of voxels in a volume of interest delimiting the location of a fluorescent bead. The observed data $\boldsymbol{y} \in \mathbb{R}^{N}$ can be modeled as

$$
(\forall n \in\{1, \ldots, N\}) \quad y_{n}=\bar{a}+\bar{b} \bar{p}_{n}+w_{n},
$$

where $\bar{a} \in \mathbb{R}$ is a background term, $\bar{b} \in] 0,+\infty[$ is a scaling parameter, $\overline{\boldsymbol{p}}=\left(\bar{p}_{n}\right)_{1 \leq n \leq N}$ represents a noiseless version of the data, and $\boldsymbol{w}=\left(w_{n}\right)_{1 \leq n \leq N} \in \mathbb{R}^{N}$ represents some acquisition noise. Our goal is to estimate $(\bar{a}, \bar{b}, \overline{\boldsymbol{p}})$ from the noisy data $\boldsymbol{y}$, assuming that $\overline{\boldsymbol{p}}$ can be well approximated by a multivariate Gaussian shape. The underlying assumption is that each entry $\bar{p}_{n}$ is close, in some sense to be precised, to the value at $\boldsymbol{x}_{n}$ of the probability density function $\boldsymbol{u} \mapsto g(\boldsymbol{u}, \overline{\boldsymbol{\mu}}, \overline{\boldsymbol{C}})$, of a $Q$-dimensional normal distribution with mean $\bar{\mu} \in \mathbb{R}^{Q}$ and precision (i.e., inverse covariance) matrix $\bar{C} \in \mathcal{S}^{++}(Q)^{1}$ expressed as

$$
\begin{gathered}
\left(\forall \boldsymbol{u} \in \mathbb{R}^{Q}\right)\left(\forall \boldsymbol{\mu} \in \mathbb{R}^{Q}\right)\left(\forall \boldsymbol{C} \in \mathcal{S}^{++}(Q)\right) \\
g(\boldsymbol{u}, \boldsymbol{\mu}, \boldsymbol{C})=\frac{|\boldsymbol{C}|^{1 / 2}}{(2 \pi)^{Q / 2}} \exp \left(-\frac{1}{2}(\boldsymbol{u}-\boldsymbol{\mu})^{\top} \boldsymbol{C}(\boldsymbol{u}-\boldsymbol{\mu})\right),
\end{gathered}
$$

where $|\boldsymbol{C}|$ denotes the determinant of matrix $C$. The problem is thus to find an estimate $(\widehat{a}, \widehat{b}, \widehat{\boldsymbol{p}}, \widehat{\boldsymbol{\mu}}, \widehat{\boldsymbol{C}})$ of $(\bar{a}, \bar{b}, \overline{\boldsymbol{p}}, \overline{\boldsymbol{\mu}}, \overline{\boldsymbol{C}})$ optimally describing the data $\boldsymbol{y}$.

Fitting Gaussian functions to multivariate empirical data is of prominent importance because of its wide array of applications in various areas such as biological imaging, but also spectroscopy, statistics and machine learning. Thus, there is a significant amount of works on this subject $[14,15,16$, 17, 18, 13]. Two classes of methods can be distinguished, either based on non-linear least squares approaches $[15,16$, $18,13]$ or on the so-called Caruana's formulation minimizing the difference of logarithms between the data and the model $[14,17,12]$. However, all the aforementioned works are focused on the resolution of the fitting problem when $Q=1$ $[14,15,17,18]$ or $Q=2[16,12,13]$. Moreover, except in [18] where a polynomial background is accounting for, the background term $\bar{a}$ is considered as zero. Finally, to the best our knowledge, all existing works consider the equality between $\boldsymbol{y}$ and its noisefree version $\bar{p}$, which may lead to a low robustness to model mismatch errors. In the next subsection,

\footnotetext{
${ }^{1}$ In the paper, $\mathcal{S}^{++}(Q)$ stands for the set of symmetric positive definite matrices of $\mathbb{R}^{Q \times Q}, \mathcal{S}^{+}(Q)$ is the set of symmetric positive semidefinite matrices of $\mathbb{R}^{Q \times Q}$ and $\mathcal{S}(Q)$ is the set of symmetric matrices of $\mathbb{R}^{Q \times Q}$.
}

we propose a novel variational approach that is able to cope with any dimension $Q$, that considers explicitly the presence of a background term, noise and that accounts for possible modeling errors in the model.

\subsection{Proposed approach}

For technical reasons, let us assume that the spectrum of $\bar{C}$ is bounded from below, i.e., there exists some $\epsilon>0$ such that we can rewrite $\overline{\boldsymbol{C}}=\overline{\boldsymbol{D}}+\epsilon \boldsymbol{I}_{Q}$ where $\overline{\boldsymbol{D}}$ belongs to $\mathcal{S}^{+}(Q)$. In practice, this simply means that the Gaussian variances in any direction are bounded above. We then propose to define $(\widehat{a}, \widehat{b}, \widehat{\boldsymbol{p}}, \widehat{\boldsymbol{\mu}}, \widehat{\boldsymbol{D}})$ as a minimizer of a hybrid cost function, gathering information regarding the observation model (1) and the Gaussian shape prior (2). In what follows, we will denote by $\iota_{S}$ the indicator function of a nonempty closed convex set $S$, so that $\iota_{S}(u)=0$ for $u \in S$, and $+\infty$ elsewhere, and $\mathcal{P}_{S}$ the projection operator on this set. This will allow us to add explicitly some constraints which are expected to be met by the sought solution. The proposed cost function reads:

$$
\begin{aligned}
& (\forall a \in \mathbb{R})(\forall b \in \mathbb{R})\left(\forall \boldsymbol{p} \in \mathbb{R}^{N}\right)\left(\forall \boldsymbol{\mu} \in \mathbb{R}^{Q}\right)(\forall \boldsymbol{D} \in \mathcal{S}(Q)) \\
& F(a, b, \boldsymbol{p}, \boldsymbol{\mu}, \boldsymbol{D})=\frac{1}{2}\|\boldsymbol{y}-a-b \boldsymbol{p}\|^{2}+\iota_{\left[a_{\min }, a_{\max }\right]}(a) \\
& +\iota_{\left[b_{\min }, b_{\max }\right]}(b)+\lambda \Psi_{\boldsymbol{X}}(\boldsymbol{p}, \boldsymbol{\mu}, \boldsymbol{D}) .
\end{aligned}
$$

In particular, $a_{\min } \leq a_{\max }$ and $0<b_{\min } \leq b_{\max }$ are some known bounds on $\bar{a}$ and $\bar{b}$ respectively, and $\lambda>0$ is a regularization parameter. The term $\Psi_{X}$ aims at introducing some prior knowledge on $\boldsymbol{p}$ and seeks for favoring the proximity between $\boldsymbol{p}$ and the Gaussian model (2) parametrized by $(\boldsymbol{\mu}, \boldsymbol{D})$. We propose to measure this closeness by the Kullback-Leibler (KL) divergence between $\boldsymbol{p}$ and the sought multivariate normal distribution [19]. Since we only have access to a discrete set of data, parametrized by their grid position $\boldsymbol{X}$, the integral usually employed in the KL divergence will be replaced by a finite summation weighted by a grid sampling step $\Delta>0$. Moreover, in order to preserve consistency of the Gaussian normalization constant, we will search for $\widehat{p}$ that belongs to the affine hyperplane $\mathcal{C}=\left\{\boldsymbol{p} \in \mathbb{R}^{N} \mid \boldsymbol{p}^{\top} \mathbf{1}_{N}=\Delta^{-1}\right\} \subset$ $\mathbb{R}^{N}$, where $\mathbf{1}_{N}$ is the vector of $\mathbb{R}^{N}$ with all entries equal to one. We also introduce the two following logarithmic functions involved in the KL term: for every $u \in \mathbb{R}$, ent $(u)$ equals $u \log u$ if $u>0,0$ if $u=0$ and $+\infty$ otherwise. For every $\boldsymbol{D} \in \mathcal{S}(Q)$, we further define

$$
\varphi(\boldsymbol{D})=\left\{\begin{array}{lr}
-\sum_{q=1}^{Q} \log \left(\sigma_{q}+\epsilon\right) & \text { if } \boldsymbol{D} \in \mathcal{S}^{+}(Q) \\
-Q \log \epsilon-\epsilon^{-1} \boldsymbol{\sigma}^{\top} \mathbf{1}_{Q}+\frac{1}{2} \epsilon^{-2} \boldsymbol{\sigma}^{\top} \boldsymbol{\sigma} & \text { otherwise, }
\end{array}\right.
$$

where $\boldsymbol{D}=\boldsymbol{U} \operatorname{Diag}(\boldsymbol{\sigma}) \boldsymbol{U}^{\top}$ with $\boldsymbol{U} \in \mathbb{R}^{Q \times Q}$ an orthogonal matrix and $\sigma=\left(\sigma_{q}\right)_{1 \leq q \leq Q}$ the associated eigenvalues. The function $\varphi$ is introduced here as a twice continuously differentiable extension of the neg-logarithmic determinant term on the whole domain $\mathcal{S}(Q)$. We are then ready to define the 
function $\Psi_{X}$ as

$$
\begin{array}{r}
\left(\forall \boldsymbol{p} \in \mathbb{R}^{N}\right)\left(\forall \boldsymbol{\mu} \in \mathbb{R}^{Q}\right)(\forall \boldsymbol{D} \in \mathcal{S}(Q)) \\
\Psi_{\boldsymbol{X}}(\boldsymbol{p}, \boldsymbol{\mu}, \boldsymbol{D})=\Delta \sum_{n=1}^{N}\left(\operatorname{ent}\left(p_{n}\right)+p_{n}\left(\frac{Q}{2} \log (2 \pi)\right.\right. \\
\left.\left.+\frac{1}{2}\left(\boldsymbol{x}_{n}-\boldsymbol{\mu}\right)^{\top}\left(\boldsymbol{D}+\epsilon \boldsymbol{I}_{Q}\right)\left(\boldsymbol{x}_{n}-\boldsymbol{\mu}\right)+\frac{1}{2} \varphi(\boldsymbol{D})\right)\right) \\
+\iota_{\mathcal{C}}(\boldsymbol{p})+\iota_{\mathcal{S}}^{+}(Q) \\
(\boldsymbol{D}) .
\end{array}
$$

\subsection{Algorithmic solution}

The objective function (3) is nonconvex, yet convex with respect to each variable. A standard resolution approach is thus to adopt an alternating minimization strategy, where, at each iteration, $F$ is minimized with respect to one variable while the others remain fixed. This approach, sometimes referred to as Block Coordinate Descent or nonlinear Gauss-Seidel method, has been widely used in the context of PSF model fitting $[13,8,11]$. However, its convergence is only guaranteed under restrictive assumptions [20]. In order to get sounder convergence results, we propose to use an alternative strategy based on proximal tools which consists of replacing, at each iteration the minimization step by a proximal step [21, 22, 23]. The application of the proximal alternating method to the minimization of (3) yields Algorithm 1, called FIGARO (Fitting Gaussians with Proximal Optimization).

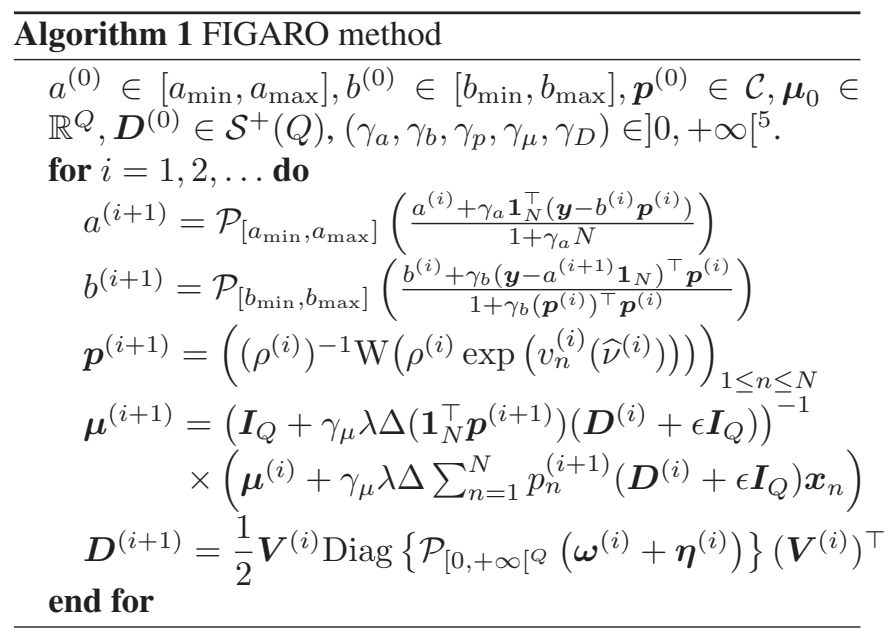

Hereabove, at each iteration $i \in \mathbb{N}, \rho^{(i)}=\left(\gamma_{p}\left(b^{(i)}\right)^{2}+\right.$ $1) /\left(\gamma_{p} \lambda \Delta\right)$, and for every $n \in\{1, \ldots, N\}, v_{n}^{(i)}: \nu \mapsto-1-$ $c^{(i)}+\left(\gamma_{p} \lambda \Delta\right)^{-1}\left(p_{n}^{(i)}+\gamma_{p} b^{(i)}\left(y_{n}-a^{(i)}\right)-\nu\right)$ with

$$
\begin{aligned}
c^{(i)}= & \frac{Q}{2} \log (2 \pi)-\frac{1}{2} \log \left(\left|\boldsymbol{D}^{(i)}+\epsilon \boldsymbol{I}_{Q}\right|\right) \\
& +\frac{1}{2} \sum_{n=1}^{N}\left(\boldsymbol{x}_{n}-\boldsymbol{\mu}^{(i)}\right)^{\top}\left(\boldsymbol{D}^{(i)}+\epsilon \boldsymbol{I}_{Q}\right)\left(\boldsymbol{x}_{n}-\boldsymbol{\mu}^{(i)}\right) .
\end{aligned}
$$

Furthermore, $\widehat{\nu}^{(i)}$ denotes the unique zero of the function $\nu \mapsto$ $\left(\rho^{(i)}\right)^{-1} \sum_{n=1}^{N} \mathrm{~W}\left(\rho^{(i)} \exp \left(v_{n}^{(i)}(\nu)\right)\right)-\Delta^{-1}$, where $\mathrm{W}$ is the Lambert-W function [24]. Finally, $\boldsymbol{\omega}^{(i)}=\left(\omega_{q}^{(i)}\right)_{1 \leq q \leq Q} \in$ $\mathbb{R}^{Q}$ (resp. $\boldsymbol{V}^{(i)} \in \mathbb{R}^{Q \times Q}$ ) is the vector of eigenvalues (resp. associated matrix of eigenvectors) of the symmetric matrix $\boldsymbol{D}^{(i)}-\frac{1}{2} \gamma_{D} \lambda \boldsymbol{S}^{(i)}$ with $\boldsymbol{S}^{(i)}=\Delta \sum_{n=1}^{N} p_{n}^{(i+1)}\left(\boldsymbol{x}_{n}-\right.$ $\left.\boldsymbol{\mu}^{(i+1)}\right)\left(\boldsymbol{x}_{n}-\boldsymbol{\mu}^{(i+1)}\right)^{\top}$, while $\boldsymbol{\eta}^{(i)} \in \mathbb{R}^{Q}$ is the vector with components $\left(\left(\left(\omega_{q}^{(i)}+\epsilon\right)^{2}+4 m^{(i)}\right)^{1 / 2}-\epsilon\right)_{1 \leq q \leq Q}$, where $m^{(i)}=\frac{1}{2} \gamma_{D} \lambda \Delta\left(\boldsymbol{p}^{(i+1)}\right)^{\top} \mathbf{1}_{N}$.

By leveraging the convergence properties of the proximal regularization of the Gauss-Seidel method algorithm [21] and the mathematical properties of (3), we were able to prove that the sequence $\left(a^{(i)}, b^{(i)}, \boldsymbol{p}^{(i)}, \boldsymbol{\mu}^{(i)}, \boldsymbol{D}^{(i)}\right)_{i \in \mathbb{N}}$ generated by FIGARO algorithm has a finite length and it converges to a critical point $(\widehat{a}, \widehat{b}, \widehat{\boldsymbol{p}}, \widehat{\boldsymbol{\mu}}, \widehat{\boldsymbol{D}})$ of the cost function $F$.

\section{APPLICATION TO TWO-PHOTON MICROSCOPY DATA}

Let us now illustrate the validity of our fitting approach in the processing of real two-photon microscopy data. Twophoton fluorescence images are obtained from a commercial multiphoton microscope dedicated to a routine use for biological multiphoton imaging (Olympus, BX61WI) with a $25 \times$ water immersion microscope objective (Olympus, XLPLN25 $\times$ WMP, 1.05 numerical aperture). The working station is coupled with a tunable femtosecond titan sapphire laser source, (Chameleon Ultra II, Coherent Inc., $800 \mathrm{~nm}$, $150 \mathrm{fs}, 10 \mathrm{~nm}, 82 \mathrm{MHz}, 30 \mathrm{~mW}$ ). The characterization of the instrument response function is tested experimentally thanks to the imaging of microbeads presenting dimensions smaller than the resolution spot. Here, spherical latex beads from Molecular Probes with a diameter of $0.2 \mu \mathrm{m}$ are involved, diluted into gelatin. It is important to emphasize that the small diameter of the beads allows us to consider each observed one as the (space-variant) instrument PSF at the bead center coordinates. 230 stacks of images of $1600 \times 1600$ squared pixels with resolution $0.053 \mu \mathrm{m}$, spaced $0.1 \mu \mathrm{m}$ apart, are acquired with FluoView FV1200 software (Olympus). Forty volumes of interest (VOIs) are selected, each of them corresponding to the noisy and blurry observation of a single bead. Figure 1 illustrates the raw acquired volume (marginalized along $\mathrm{Y}$ axis) and the position of selected VOIs.

We then apply the FIGARO method to fit a 3D Gaussian model to each of them. Our processing method does not require any user intervention. In particular, an efficient $\chi^{2}$ strategy is employed for automatically setting the regularization parameter $\lambda$ [25]. An example of 3D fitting result is displayed in Fig. 2 (left). We also present in Table 1 some numerical results obtained for the estimation of the center coordinates, full width half maximum (FWHM), and orientation (Euler angles) of the fitted Gaussian shapes, using our method, and the 


\begin{tabular}{|c|l|c|c|c|c|}
\hline \multicolumn{2}{|c|}{ Volume of Interest } & $\mathrm{n}^{\circ} 1$ & $\mathrm{n}^{\circ} 2$ & $\mathrm{n}^{\circ} 3$ & $\mathrm{n}^{\circ} 4$ \\
\hline \hline \multirow{2}{*}{ MetroloJ } & Center $(\mu \mathrm{m})$ & $(62.77,18.59,5.46)$ & $(41.62,65.69,5.50)$ & $(66.01,0.35,13.82)$ & $(10.24,66.96,10.46)$ \\
& FWHM $(\mu \mathrm{m})$ & $(0.32,0.03,0.05)$ & $(0.29,0.03,0.001)$ & $(0.028,0.19,0.1)$ & $(0.05,0.04,0.57)$ \\
\hline \multirow{3}{*}{ FIGARO } & Center $(\mu \mathrm{m})$ & $(62.78,19.19,7.57)$ & $(41.71,66.27,6.10)$ & $(66.22,1.03,14.61)$ & $(10.29,67.59,11.72)$ \\
& FWHM $(\mu \mathrm{m})$ & $(0.192,0.247,1.275)$ & $(0.201,0.307,1.282)$ & $(0.198,0.252,1.539)$ & $(0.205,0.259,1.601)$ \\
& Angles $\left({ }^{\circ}\right)$ & $(73.1,2.38)$ & $(67.3,5.63)$ & $(87.2,1.54)$ & $(105.6,2.24)$ \\
\hline
\end{tabular}

Table 1. Example of fitting results on 4 VOIs for our approach, and the MetroloJ plugin from Fiji.

MetroloJ plugin of Fiji ${ }^{2}$. The latter does not allow to estimate the orientation as it relies on a 1D Gaussian fitting on the marginals along each 3 axis. The estimated center positions are quite similar for both methods, mainly because of the small size of the VOIs. The high variability of the MetroloJ results in terms of FWHM emphasizes the importance of being able to deal directly with 3D models, as well as the difficulty of coping with such high noise level. In contrast, our method appears to be very robust to noise, as it allows to estimate precisely the width and the orientation axis of the PSF in the 3D space. An analysis of our results for the whole set of VOIs shows that, for this dataset and this range of depths, the planar width of the PSFs does not vary much with respect to the beads location. Here, the averaged FWHM of the estimated Gaussian shapes is of $(0.21,0.27) \mu \mathrm{m}$ in the $\mathrm{XY}$ plane, which appears to be consistent with the theoretical limit of optical planar resolution of $0.2 \mu \mathrm{m}$ for this emission wavelength and numerical aperture. The axial PSF width is slightly increasing when the depth of the bead center increases (Fig. 2 (right)), as it is expected from the optical theory [26], and the averaged axial resolution is of $1.49 \mu \mathrm{m}$ which fits well with the theoretical resolution limit of $1.5 \mu \mathrm{m}$ displayed in the literature [27]. The PSFs orientations we are measuring change according to the beads location, and the tilt angle (i.e., angle between principal eigenvector and $Z$ axis) varies within a range of $[0.6,7.7]^{\circ}$ for this dataset. A standard analysis based on 1D (or even 2D) fitting cannot have access to such a precise estimation of the tilt angle, yet of main importance for an efficient processing of the microscopic images.

In order to highlight the impact of our results, we have completed our analysis with a step of deblurring of a subpart of the whole dataset, with size $200 \times 200 \times 50$ voxels, corresponding to a field of view of $10 \times 10 \times 5 \mu \mathrm{m}$. A constant PSF was considered in this region, whose width and orientation are deduced from our fitting results by interpolation. The deblurring step is performed using the OPTIMISM toolbox from Fiji ${ }^{3}$ [28]. Figure 3 illustrates one slice extracted from the input and restored images. One can observe a high improvement in terms of resolution, which highlights the practical interest of our PSF fitting approach.

\footnotetext{
${ }^{2}$ http://imagejdocu.tudor.lu/doku.php?id=plugin:analysis:metroloj:start ${ }^{3} \mathrm{http}: / /$ sites.imagej.net/Dbenielli/
}

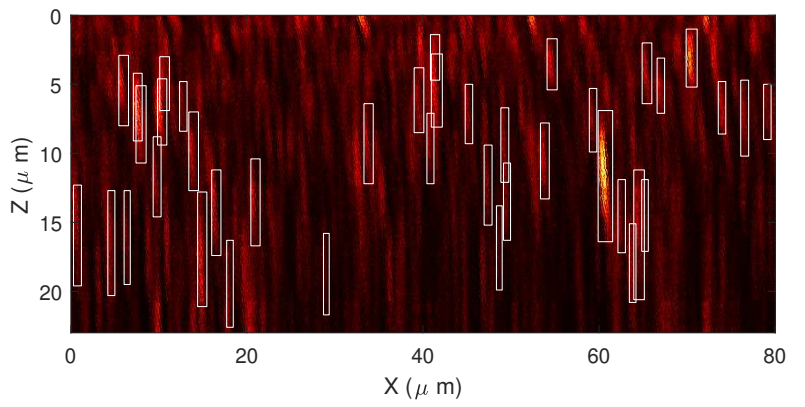

Fig. 1. Input data and locations of VOIs represented by white boxes.
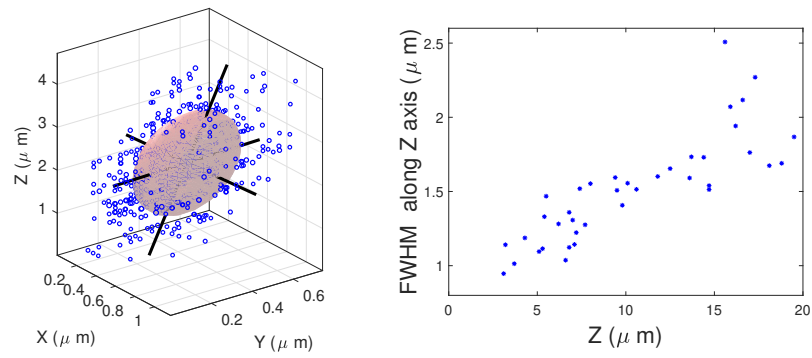

Fig. 2. Left: Example of 3D fitting result. Right: Evolution of the estimated axial FWHM with the bead center depth.
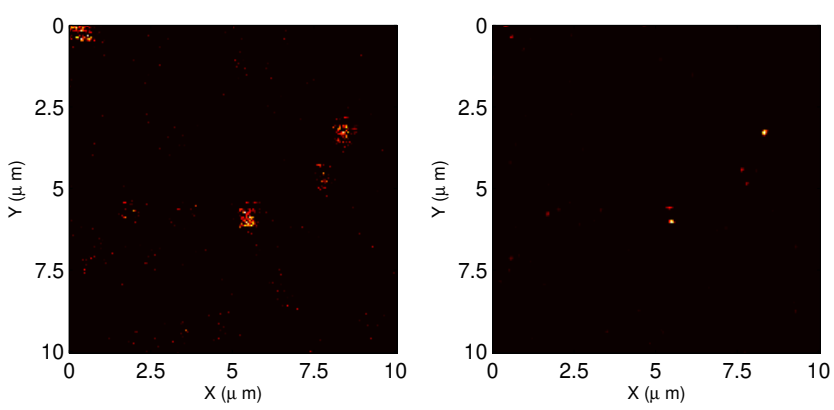

Fig. 3. Deblurring results: Slice 32 of input data (left) and restored volume (right).

\section{CONCLUSION}

In this paper, a novel optimization approach called FIGARO has been introduced for multivariate Gaussian shape fitting, with guaranteed convergence properties. Experiments have clearly illustrated the applicative interest of FIGARO, in the context of PSF identification in two-photon imaging. The versatility of FIGARO makes it applicable to a wide range of applicative areas, in particular other microscopy modalities. 


\section{REFERENCES}

[1] W. Denk, J. H. Strickler, and W. W. Webb, “Two-photon laser scanning fluorescence microscopy," Science, vol. 248, no. 4951, pp. 73-76, 1990.

[2] M. Göppert-Mayer, "Über elementarakte mit zwei quantensprüngen,” Ann. Phys., vol. 401, no. 3, pp. 273-294, 1931.

[3] C. Lefort, "A review of biomedical multiphoton microscopy and its laser sources," J. Phys. D: Appl. Phys., vol. 50, no. 42, pp. 423001, 2017.

[4] S. W. Hell et al., "The 2015 super-resolution microscopy roadmap,” J. Phys. D: Appl. Phys., vol. 48, no. 44, pp. 443001, 2015.

[5] E. Chouzenoux, A. Jezierska, J.-C. Pesquet, and H. Talbot, "A convex approach for image restoration with exact PoissonGaussian likelihood," SIAM J. Imag. Sci., vol. 8, no. 4, pp. 2662-2682, 2015.

[6] A. Jezierska, Image Restoration in the presence of PoissonGaussian noise, Ph.D. thesis, University Paris East Marne-laVallée, 2013.

[7] H. Kirshner, T. Pengo, N. Olivier, D. Sage, S. Manley, and M. Unser, "A PSF-based approach to biplane calibration in 3D super-resolution microscopy," in 9th IEEE Int. Symp. Biomed. Imaging (ISBI 2012), Barcelona, Spain, 2-5 May 2012, pp. 1232-1235.

[8] H. Kirshner, F. Ahuet, D. Sage, and M. Unser, "3-D PSF fitting for fluorescence microscopy: implementation and localization application," J. Microsc., vol. 249, no. 1, pp. 13-25, 2013.

[9] B. Zhang, J. Zerubia, and J.-C. Olivo-Marin, "Gaussian approximations of fluorescence microscope point-spread function models," Appl. Opt., vol. 46, no. 10, pp. 1819-1829, Apr 2007.

[10] P. Sarder and A. Nehorai, "Estimating locations of quantumdot-encoded microparticles from ultra-high density 3-D microarrays," IEEE Trans. NanoBioscience, vol. 7, no. 4, pp. 284-297, Dec. 2008.

[11] M. Marim, B. Zhang, J. C. Olivo-Marin, and C. Zimmer, "Improving single particle localization with an empirically calibrated Gaussian kernel," in 5th IEEE Int. Symp. Biomed. Imag.: From Nano to Macro (ISBI 2008), Paris, France, 14-17 May 2008, pp. 1003-1006.

[12] S. M. Anthony and S. Granick, "Image analysis with rapid and accurate two-dimensional Gaussian fitting," Langmuir, vol. 25, no. 14, pp. 8152-8160, 2009.

[13] X. Zhu and D. Zhang, "Efficient parallel Levenberg-Marquardt model fitting towards real-time automated parametric imaging microscopy," PLOS ONE, vol. 8, no. 10, pp. 1-9, 102013.

[14] R. Caruana, R. Searle, T. Heller, and S. Shupack, "Fast algorithm for the resolution of spectra," Anal. Chem., vol. 58, no. 6, pp. 1162-1167, May 1986.

[15] N. Hagen, M. Kupinski, and E. L. Dereniak, "Gaussian profile estimation in one dimension," Appl. Opt., vol. 46, no. 22, pp. 5374-5383, Aug 2007.
[16] N. Hagen and E. L. Dereniak, "Gaussian profile estimation in two dimensions," Appl. Opt., vol. 47, no. 36, pp. 6842-6851, Dec 2008.

[17] H. Guo, "A simple algorithm for fitting a Gaussian function [DSP tips and tricks]," IEEE Signal Proc. Mag., vol. 28, no. 5, pp. 134-137, Sept 2011.

[18] E. K. Roonizi, "A new algorithm for fitting a Gaussian function riding on the polynomial background," IEEE Signal Process. Lett., vol. 20, no. 11, pp. 1062-1065, Nov 2013.

[19] M. Basseville and J. F. Cardoso, “On entropies, divergences, and mean values," in IEEE Int. Symp. Inf. Theory (ISIT 1995), Whistler, BC, 1722 Sep. 1995, p. 330.

[20] P. Tseng, "Convergence of a block coordinate descent method for nondifferentiable minimization," Journal of Optimization Theory and Applications, vol. 109, no. 3, pp. 475-494, 2001.

[21] H. Attouch, J. Bolte, and B. F. Svaiter, "Convergence of descent methods for semi-algebraic and tame problems: proximal algorithms, forward-backward splitting, and regularized Gauss-Seidel methods," Math. Prog., Ser. A, vol. 137, no. 1, pp. 91-129, 2013.

[22] H. Attouch, J. Bolte, P. Redont, and A. Soubeyran, "Proximal alternating minimization and projection methods for nonconvex problems: an approach based on the Kurdyka-Lojasiewicz inequality," Mathematics of Operations Research, vol. 35, no. 2, pp. 438-457, 2010.

[23] J. Bolte, P. L. Combettes, and J. C. Pesquet, "Alternating proximal algorithm for blind image recovery," in Proc. IEEE Int. Conf. Image Process. (ICIP 2010), Hong-Kong, China, 26-29 Sept. 2010, pp. 1673-1676.

[24] R. M. Corless, G. H. Gonnet, D. E. G. Hare, D. J. Jeffrey, and D. E. Knuth, "On the Lambert $W$ function," $A d v$. Comput. Math., vol. 5, no. 1, pp. 329-359, 1996.

[25] N. P. Galatsanos and A. K. Katsaggelos, "Methods for choosing the regularization parameter and estimating the noise variance in image restoration and their relation," IEEE Trans. Image Process., vol. 1, no. 3, pp. 322-336, Jul 1992.

[26] F. Helmchen and W. Denk, "Deep tissue two-photon microscopy," Nat. Methods, vol. 2, no. 12, 2005.

[27] E. Tal, D. Oron, and Y. Silberberg, "Improved depth resolution in video-rate line-scanning multiphoton microscopy using temporal focusing," Opt. Lett., vol. 30, no. 13, pp. 1686-1688, 2005.

[28] E. Chouzenoux, L. Lamassé, C. Chaux, A. Jaouen, I. Vanzetta, and F. Debarbieux, "Approche variationnelle pour la déconvolution rapide de données $3 \mathrm{~d}$ en microscopie biphotonique," in Actes du 25e colloque GRETSI, 8-11 Sep. 2015. 